\title{
Desigualdades socioecológicas y sufrimiento ambiental en el conflicto "Polimetales" en Arica
}

\author{
Socio-ecological inequalities and environmental suffering \\ in the "Polimetales" conflict in Arica
}

\section{Mayarí Castillo-Gallardo / mcastillog@docentes.academia.cl Universidad Academia de Humanismo Cristiano, Chile}

\begin{abstract}
This article explores the cultural construction of socio-ecological inequalities, recovering the concept of environmental suffering for the analysis of a territory with high environmental degradation: the "Polimetales" case located in the city of Arica, Chile, studied through an ethnographic methodology during 2013, 2014 and 2015. The paper attempts to establish the ways in which the subjects involved in these territories experience this environmental suffering, interpret socio-environmental unequal relationships and try to modify them. By means of this analysis, the article focus on the dynamics of conflict about inequalities.

Key words: socio-ecological inequalities, environmental suffering, Arica, Chile, conflict.

Resumen: Este artículo indaga en la construcción cultural de las desigualdades socioecológicas, recuperando el concepto de sufrimiento ambiental para el análisis de un territorio de alta degradación ambiental: el caso "Polimetales", ubicado en la ciudad de Arica, estudiado a través de una metodología etnográfica durante los años 2013, 2014 y 2015. Se busca establecer las formas mediante las cuales los sujetos involucrados en estos territorios padecen dicho sufrimiento ambiental, interpretan las relaciones de desigualdad socioambiental en las que se encuentran, intentan modificarlas o muchas veces las reproducen. Con este análisis, el artículo reflexiona sobre las dinámicas de conflicto en torno a las desigualdades.
\end{abstract}

Palabras clave: desigualdades socioecológicas, sufrimiento ambiental, Chile, Arica, conflicto. 


\section{Introducción ${ }^{1}$}

Es poca la investigación sobre desigualdad que se ha producido en las últimas décadas desde una mirada cualitativa y sobre aquellas dimensiones socioculturales asociadas. Al parecer, hoy las ciencias sociales de América Latina han olvidado que las sociedades desiguales estructuran la experiencia cotidiana y percepciones de los individuos, articulándose con complejos entramados culturales e institucionales (Tilly, 2000; Reygadas, 2004; 2008a; 2008b).

Este artículo busca ser un aporte en esta dirección de interpretación, enfocándose en aspectos relacionados con la construcción cultural de la desigualdad en las sociedades contemporáneas, específicamente en la sociedad chilena.

Para un análisis desde este enfoque, el artículo indaga en la construcción cultural de uno de los fenómenos que ha encontrado escaso desarrollo en la agenda de investigación de estudios sobre desigualdad en las últimas décadas, aunque no ha dejado de estar presente de alguna manera en la reflexión desde el campo de la geografía o la ecología política (Harvey, 1996; Smith, 2008; Müller y Clayton, 2013): las desigualdades socioecológicas.

Rescatando algunos aportes de esta tradición, se parte de la hipótesis de que al igual que otros bienes socialmente valorados, en sociedades desiguales el vivir en un ambiente libre de contaminación es también un bien que encuentra una distribución desigual. Las variables más influyentes en que los sujetos se vean expuestos a vivir en un territorio degradado ambientalmente y con altos niveles de contaminación son las mismas que determinan su posición en términos de acceso a otros bienes: la pertenencia étnica/racial, de clase, género y territorial, por nombrar las más relevantes para el caso chileno.

Esto ha sido considerado por quienes han trabajado con los conceptos de racismo ambiental y justicia ambiental a partir de la década de 1970 (Bullard, 1990; 1999; 2005; Dosbon, 1998; Pezullo, 2001; Checker, 2005; Cole, 2005; Harvey, 1996; Smith, 2008; Müller y Clayton, 2013) y ha sido señalado también para el caso latinoamericano en la última década (Guimarães, 2012; Auyero y Swistún, 2008; Vázquez y Salgado, 2009; Castillo, 2015).

En el caso chileno, esto resulta notorio si analizamos la composición en términos socioeconómicos en la que se concentran algunas actividades económicas: sin ir más lejos, en la ciudad de Santiago las comunas donde se reúnen las principales actividades industriales son aquellas donde el porcentaje de

1 Investigación financiada por: Proyecto FONDECYT de Iniciación núm. 11140008 y Centro Interdisciplinario de Estudios Interculturales e Indígenas-CIIR, Código de Proyecto: CONICYT/FONDAP/ núm. 15110006. 
población perteneciente a grupos ocupacionales en la base de la estratificación social supera el $50 \%$.

Tal es el caso de Renca con $66,2 \%$, Cerro Navia con 71,4\%, Cerrillos con $61.1 \%$ y Pudahuel con $57,3 \%$, por nombrar los polos industriales más relevantes y con mayor cantidad de problemas de externalidades negativas para sus vecinos. ${ }^{2}$ Parafraseando a Auyero (2008), es posible decir entonces que los sujetos vulnerables no sólo tienen problemas relativos a ingresos o condiciones de trabajo desiguales, sino también se ven expuestos a mayores riesgos ambientales y a enfermedades derivadas de éstos que quienes se encuentran en posiciones de mayor privilegio.

Los efectos de la contaminación y degradación ambiental se ven agravados en dichas circunstancias, pues la exposición tóxica es un elemento más dentro de un contexto de vulnerabilidad general que dificulta los procesos de acción/ reacción/protección de los sujetos, generando situaciones bastante específicas de sufrimiento social, incertidumbre y contextos de crisis que reproducen muchas veces las relaciones de dominación presentes en los territorios.

Para analizar este fenómeno a nivel sociocultural, el artículo recupera el concepto de sufrimiento ambiental utilizado por Auyero y Swintún (2008) en el análisis del caso "Inflamable" en Argentina. En dicho estudio, los autores proponen la noción de sufrimiento ambiental como concepto que agrupa la experiencia de vivir en un contexto tóxico y que sobrepasa los meros efectos biológicos. Incorporan así todos aquellos elementos que van configurando las posiciones de desigualdad, directamente relacionados con la exposición tóxica en la que se encuentran los sujetos, la lentitud de las soluciones y la escasa visibilidad pública del conflicto.

Con esta noción Auyero y Swintún (2008) destacan que hay una violencia invisible que se impone sobre quienes son expuestos a contextos tóxicos: no sólo porque hay una serie de variables que determinan que vivan en estos territorios y se vean expuestos, sino también porque dichas variables influyen en nuevas formas de victimización en el marco de la exposición, como malos tratos en el sistema de salud, invisibilidad de sus demandas en el espacio público,

2 El enfoque con el cual se trabajaron los datos de la Encuesta de Caracterización Socioeconómica Nacional 2006 (Ministerio de Desarrollo Social, Gobierno de Chile, 2006) fue el análisis de clases, basado en el trabajo de E. O. Wright (1985; 2009), a partir de cuyo esquema y con base en los grandes grupos del código CIUO se establecieron segmentos de clase. A partir éstos, se clasificaron las comunas del Gran Santiago de acuerdo con la proporción de jefes de hogar económicamente activos que pertenecían a las distintas categorías de clase, considerando el peso relativo que estas clases poseían en el total de la población. 
soluciones deficientes al problema de la contaminación, reubicaciones no consensuadas, por nombrar algunas de las más comunes, que revisaré más adelante en la exposición del caso analizado.

Así, se utiliza este concepto para el análisis de un territorio de alta degradación ambiental: el caso "Polimetales", ubicado en la ciudad de Arica, ${ }^{3}$ estudiado durante los años 2013, 2014 y 2015. Se busca establecer las formas mediante las cuales los sujetos involucrados en estos territorios padecen dicho sufrimiento ambiental, interpretan las relaciones de desigualdad socioambiental en las que se encuentran, intentan modificarlas o muchas veces las reproducen. Aquí, los sujetos se hallan constantemente interpelados por una degradación ambiental visible -e invisible en el caso de los efectos silenciosos, de los cuales sospechan los habitantes- y van elaborando relatos con una “Gramática de la Desigualdad” (Boltanski y Chiapello, 2002) que los orienta.

Para analizar este proceso, se utilizan los aportes de los enfoques teóricos recientemente desarrollados sobre el tema (Boltanski y Thévenot, 1999; Boltanski y Chiapello, 2002; Boltanski et al., 2006; Thévenot et al., 2011), que rescatan la necesidad de observar la dimensión cultural de las desigualdades y el rol de los sujetos en la transformación de ésta. Para estos autores, las políticas redistributivas no tienen un efecto en sí mismo, sino en tanto son capaces de modificar patrones culturales persistentes, que permean la cotidianidad de los sujetos y que van estructurando mecanismos de reproducción de la desigualdad, hecho que también ha sido señalado en la última década para el caso latinoamericano, aunque de manera incipiente (Gootenbergy Reygadas, 2010; Castillo y Maldonado, 2015).

Dentro de los elementos teóricos a considerar de estos enfoques, cabe destacar el rol de activo del sujeto en la transformación de dichas estructuras a partir de la crítica permanente y el rol del conflicto en estos procesos, en donde resultan claves las modificaciones que se observan en los discursos y significados que articulan la crítica e impulsan la conflictividad en las sociedades contemporáneas.

En esos términos, resulta central el análisis de los relatos que los sujetos elaboran en estas posiciones de extrema vulnerabilidad y que se encuentran en el corazón de las dinámicas de conflicto en torno a las desigualdades, ya que la emergencia de molestia, reclamo o conflictividad respecto a las nociones de riesgo y contaminación requiere un complejo proceso a través del cual los sujetos van transformando lo que les parece "normal" o "injusto", así como se van modificando también las percepciones y expectativas sobre medio ambiente, "calidad de vida", "limpio" y "sucio".

3 La ciudad de Arica se encuentra en el norte de Chile, en la zona fronteriza con Perú y Bolivia. 
La emergencia de conflictividad en estos contextos va unida a procesos de construcción de sentido, por un lado, y a un proceso de constitución y reconocimiento del sujeto "contaminado", por el otro. Este último proceso resulta particularmente interesante porque va transformando las subjetividades en el marco del conflicto, impulsando procesos de empoderamiento y articulaciones identitarias nuevas.

Dicho proceso tiene también una dimensión altamente violenta, ya que las comunidades deben pelear por la legitimidad de su dolor y por la importancia de la reparación frente a otro que muchas veces los niega: en ese tránsito, los efectos sobre el cuerpo son escrutados, los espacios más privados son expuestos y los lugares colectivos son transformados en ejemplos del daño ocasionado.

El logro del reconocimiento del "sujeto contaminado" va dejando huellas dolorosas que liga las biografías individuales y familiares a las ideas de daño y enfermedad; al mismo tiempo, los sujetos deben cargar con la estigmatización por habitar estos espacios, aunada muchas veces a la que ya suelen padecer por ser población vulnerable.

Con el fin de dar cuenta de estos procesos, se presentan los resultados del análisis del caso del conflicto por contaminación por polimetales en barrios periféricos de la fronteriza ciudad de Arica, emplazada en el norte de Chile. Para este caso se ocupó el método etnográfico (Baztan, 1997), enfatizando la documentación de aspectos relativos a la cotidianidad de la experiencia tóxica.

Durante los tres años que se ha llevado a cabo la investigación, se han aplicado técnicas como la entrevista semiestructurada, la observación participante, el análisis documental y de prensa, entre otras. Respecto a las entrevistas, se han realizado 40 a actores claves en el territorio, segmentados en cuatro grupos: $a)$ dirigentes de organizaciones sociales y territoriales; $b$ ) autoridades político-administrativas involucradas; c) funcionarios públicos en contacto con la población del territorio (salud, educación y dirección comunitaria); y d) expertos/abogados y ONGS.

A la vez, se han recopilado un total de 13 causas judiciales y abundante material fotográfico, documental y archivos de prensa local y nacional. Parte de estos materiales han sido obtenidos por el equipo de la investigación aquí presentada. Otros han sido facilitados por los mismos afectados durante el transcurso de las distintas visitas a terreno que se han realizado. La importancia de la colaboración de los habitantes de los territorios en conflicto habla de una inmensa necesidad de hacerse visibles en la esfera pública, con el fin de encontrar alguna reparación, hasta ahora sin resolver. 
Dado el gran volumen de información, los materiales fueron examinados a través de análisis de contenido bottom up, asistido a través del software para análisis cualitativo Nvivo. A continuación se exponen los resultados preliminares del presente estudio de caso de larga historicidad, razón por la cual se exhiben de manera sintética y organizada en función de ciertos hitos demarcatorios que integran la distinta evidencia empírica recopilada, permitiendo la comprensión de las transformaciones que ha operado en torno a la "gramática de la desigualdad" (Boltanski y Thévenot, 1999) en el caso y su relación con ciertas dinámicas del conflicto en el territorio.

\section{Arica. El caso "Polimetales" y la búsqueda de reconocimiento}

El caso analizado se encuentra emplazado en las poblaciones de Cerro Chuño y Los Industriales I, II, III, IV, ubicados en la periferia de la ciudad fronteriza de Arica. En estos territorios se ha documentado contaminación por múltiples metales pesados - plomo y arsénico, principalmente- en zonas habitadas por población pobre y vulnerable. Las primeras acciones del caso se registraron a principios de la década de 1990 y hoy, casi treinta años después, el conflicto se mantiene vigente, experimentando una creciente judicialización a partir del año 2014.

La presencia de metales proviene de la utilización de la zona como lugar de acopio de desechos con altos contenidos de plomo y arsénico provenientes de Suecia e ingresados al país durante 1984 y 1985 por la empresa Procesadora de Metales Ltda. (Promel), en forma de materiales destinados a la extracción de metales como el oro y la plata. El ingreso -se ha referido en varias oportunidades por canales oficiales- tenía por objetivo el procesamiento de estos desechos como actividad productiva a cargo de dicha empresa chilena.

Sin embargo, los hallazgos de las primeras acciones judiciales emprendidas por los afectados han mostrado que los socios europeos de Promel habrían declarado abiertamente su intención de establecer un trato comercial en torno a la eliminación de desechos tóxicos no permitidos en Suecia y que éstos no estarían en condiciones de formar parte de una nueva cadena productiva.

Estos elementos se encuentran aún en disputa por parte de los distintos actores: las diferentes versiones respecto al tema son sintomáticas de la confusión e incertidumbre generalizada sobre éste. Lo que sí sabemos es que casi cuatro años después de la internación de los residuos, el emplazamiento de la empresa Promel cerró sus puertas y desmanteló sus instalaciones sin explicación alguna, despidiendo a sus trabajadores y desarmando la planta. 
Posterior al cierre de esta empresa, en la década de 1990, en el espacio contaminado por las actividades de Promel hubo procesos de autoconstrucción y "tomas de terreno". Pero en su mayoría fue utilizado por el Gobierno Regional y el Ministerio de Vivienda para ubicar hogares destinados a la población vulnerable y pobre, asignados mediante el sistema de subsidio a la vivienda social. De manera adicional, en las inmediaciones de la zona residencial de Cerro Chuño se emplazó el vertedero municipal de la ciudad, actualmente en uso.

Estas hogares fueron habitados por la población durante un periodo breve antes de que ésta empezara a manifestar síntomas de envenenamiento por plomo y arsénico; ${ }^{4}$ dicha situación fue denunciada sin éxito por diversos actores locales por casi una década. Durante este proceso y a pesar de ciertas medidas cautelares por parte del Servicio Regional Ministerial de Salud (SEREMI) en el año 1997, se finalizó la construcción y entrega de nuevas viviendas sociales en la zona.

Tras casi veinte años de conflicto, en el año 2009, por primera vez, hubo un reconocimiento oficial de la gravedad de la situación en el documento principal del Programa Maestro de Intervención en Zonas con presencia de Polimetales en Arica (de ahora en adelante "Plan Maestro").

Este Plan Maestro, además de reconocer oficialmente el problema, indicó una serie de medidas de intervención en el territorio. En 2012, y luego de un intenso conflicto, el "Plan Maestro" se convirtió en ley y entró en ejecución en 2013. ${ }^{5}$ En este proceso, el proyecto de ley sufrió numerosas modificaciones y su resultado final no fue aprobado por las organizaciones involucradas, las que hasta el día de hoy siguen apelando a la implementación del "Plan Maestro" originalmente pactado con la autoridad de la época, la entonces ministra de Salud Michelle Bachelet.

Cuando se concluyó la elaboración de este artículo, existía todavía un intenso debate en la zona, en torno a las políticas reparatorias y en función de su ejecución. Pese a lo largo y complejo de este conflicto, se pueden distinguir

4 Entre éstos figuran síntomas como alergias, dolores de cabeza, pérdidas de embarazos de estado avanzado, malformaciones en fetos y recién nacidos, problemas en articulaciones y huesos, problemas de concentración y en el funcionamiento de órganos blandos.

5 Entre las medidas consideradas en la ley se decretó la incorporación de los afectados a un plan de seguimiento en salud, becas a nivel universitario y un apoyo monetario mensual para quienes acreditasen su condición de "exposición”. También se modificaron los territorios por reubicar según lo establecido originalmente en el "Plan Maestro". Para más detalles, véase ley núm. 20.590, Programa de Intervención en Zonas con Presencia de Polimetales en La Comuna de Arica. 
varios hitos referenciales que impulsaron acciones concretas por parte de los vecinos y que modificaron la forma de percibir la cotidianidad y fueron moldeando la experiencia tóxica.

En cada uno de ellos se pueden ver reflejadas las diferencias de visibilidad pública y las dinámicas de dominación, poder y conflicto entre los actores del territorio, marcadas por la condición de extrema vulnerabilidad de la población afectada. Este caso nos permite observar claramente la relación entre experiencia tóxica, las posiciones desiguales de los afectados frente a los diferentes actores y percepción del conflicto, que se va modificando a lo largo de tiempo a partir de elementos como la voz de los expertos, la voz de la institucionalidad y la voz de los medios de comunicación, por nombrar los más relevantes.

Esto nos posibilita reflexionar también en torno a la condición de invisibles de ciertos sectores de la población en el ámbito de nuestras democracias y sobre la necesidad de generar escenarios de igualdad que permitan que estos sectores sean "vistos y oídos" en el marco de un debate público, rescatando el sentido del espacio político planteado por Arendt (Castillo, 2013).

\section{Medicina y poder. Estar enfermo para ser "visible"}

Sin duda uno de los elementos que llaman la atención de este caso son los niveles desiguales de visibilidad pública que cruzan la trayectoria del conflicto y están marcados por la situación de pobreza y extrema pobreza de los beneficiarios de las viviendas sociales construidas en el polígono contaminado.

Luego de habitar las viviendas durante un corto periodo, la población afectada manifiesta una serie de síntomas físicos, cuya relación con el problema de los residuos tóxicos es completamente ignorada tanto por ellos mismos como por el personal de salud en contacto directo con ella. La problemática se detona cuando las organizaciones vecinales -impulsadas por agentes territoriales y municipales-, la Oficina de Medio Ambiente y Secretaría de Planificación de la Municipalidad (SECPLAN) y ONG medio ambientales realizan un primer diagnóstico para identificar en general los problemas de la población asociados con la pobreza y la vulnerabilidad.

Los resultados de este diagnóstico dirigen las sospechas a un "montículo" ubicado en el centro del perímetro habitado y que enciende las alarmas de ONG y ambientalistas, que toman muestras independientes de suelo y polvo de los techos, con resultados positivos para altos niveles de plomo. Frente a la denuncia de esta situación, las autoridades locales y regionales no tuvieron reacción alguna. 
Durante este periodo, los habitantes acudían a los centros asistenciales de salud del territorio y eran remitidos de vuelta a sus casas con medidas orientadas al control de síntomas, sin mayores indagaciones. En muchas ocasiones los entrevistados refieren que se desestimaban sus sospechas en las enfermedades de los niños, aludiendo a un mal cuidado, falta de aseo por parte de las madres o negligencia.

En estos testimonios se puede ver la violencia que se impone sobre alguien "invisible" a los ojos del funcionario municipal en su dolencia y espacio privado mínimo: su cuerpo. Tal como se constata en la siguiente cita, durante estos tratamientos iniciales, los funcionarios interpretaron y actuaron frente a la situación a partir de una peligrosa asociación entre pobreza, suciedad, enfermedad y negligencia, que impidió en un primer momento una atención oportuna:

Los niños iban a jugar ahí y les empezaron a salir ronchas, como sarna, obviamente empezaron a tener reacciones y cuando empezaron a llevar a los niños al médico, los médicos decían que los niños tenían sarna y que era porque éramos $\operatorname{cochinos}^{6}$ (Dirigente territorial, mujer, marzo de 2014).

Pese a las denuncias de las organizaciones vecinales y ONG asociadas -aún escasas en número-, no se emprendieron mayores acciones y tampoco se paralizó la entrega de viviendas en la zona. Los vecinos -excepto los dirigentes vinculados con redes y organizaciones fuera del territorio- no estaban informados de lo sucedido o desestimaban las denuncias y su importancia. Hasta ese momento no había "pruebas" de nada, pese a las tomas de muestra de suelo y techos de las ONG de la zona. Uno de los elementos fundamentales que influía en desestimar el problema entre los vecinos era la constante negación de los centros de salud en contacto con la población.

El primer hito referencial que modificó esta percepción -para autoridades y habitantes- fue el establecimiento formal del envenenamiento por metales pesados a través de pruebas médicas en la población expuesta, que tuvo lugar luego de una serie de acciones por parte de vecinos y ONG. Ésta se emprendió en 1998 cuando los organismos de salud competentes se vieron obligados a realizar análisis de sangre a la gente.

Los resultados arrojaron niveles de plomo en sangre y de arsénico inorgánico en orina mayores a los referenciales por la Organización Mundial de la Salud (2012) sobre todo en los niños ${ }^{7}$ y determinaron el traslado del

6 Término coloquial: sucios.

7 Los niveles de referencia OMS son de un máximo de $10 \mathrm{lg} / \mathrm{l}$ y $25 \mathrm{lg} / \mathrm{dl}$ para plomo y arsénico, respectivamente. Para mayor información, véase: http://www.who.int/topics/ arsenic/es/ 
"Montículo" de residuos a la zona de la Quebrada Encantada, a menos de cinco kilómetros del espacio habitado. Pero antes de decretar esta medida, la "toma de muestras de sangre" se vio empañada por la desacertada acción de los organismos de salud, que -dice la población como un secreto a vocesadulteraron una parte de las muestras y extraviaron otra, desatando la furia de los dirigentes y la desconfianza de la gente. Las organizaciones (Juntas de vecinos de las zonas afectadas y las 14 dirigentas del plomo) denunciaron públicamente esta situación a través de la prensa, y las autoridades les ofrecieron disculpas.

La ratificación, mediante exámenes, de los niveles de contaminación, así como su extravío y adulteración, resultaron clave para la percepción de los habitantes y las posteriores acciones realizadas por las organizaciones. Antes de que esta medición tuviera lugar, la efectiva contaminación en la zona era todavía un tema no establecido ni "creíble". A la ratificación de los altos niveles de metales se le sumó la certeza creciente de que si las autoridades actuaban de manera tan poco transparente es porque "algo hay" o "por algo lo hacen".

Contrario al efecto esperado, el comportamiento de los organismos de salud apuntaló las dudas de gran parte de la población y les permitió establecer una relación en términos de percepción-comprobable o no-entre la contaminación y una serie de síntomas físicos y psicológicos que veían en sus propios cuerpos y los de sus familias.

Así, la "toma de muestras" modificó de una vez y para siempre la percepción de los habitantes sobre su entorno, su casa y su espacio común. Los lugares que antes eran sitios de juego se volvieron espacios prohibidos y el polvo de las casas era continuamente limpiado. En este punto, los sujetos comenzaron a padecer el sufrimiento que implica no saber a ciencia cierta los efectos que los desechos tóxicos tenían sobre su salud, a sentirse vulnerables por la acción abusiva de las autoridades y a sentir miedo por perder lo invertido en el mejoramiento de sus ansiadas casas.

Este sufrimiento, tal y como lo documentan Auyero y Swistún (2008) en el caso de "Inflamable", está estrechamente vinculado con la incertidumbre y con los niveles de información desigual y contradictoria a la que se ven expuestos los habitantes de las zonas contaminadas, que se acrecienta por la vulnerabilidad por las variables previas a la exposición. Estas variables previas los hace, en términos de Chaterjee (2008) - “objetos de la política pública”-, depositarios de la acción de un Estado que los mantiene en una permanente espera y que maneja información clave para entender la propia experiencia y entorno. 
En ese sentido, el conocimiento experto se vuelve central como elemento que da forma a la experiencia tóxica para los habitantes, a la vez que legitima la demanda de éstos en el espacio público: la voz de dichos actores locales se vuelve relevante sólo si existe una institucionalidad que, a través de las pruebas médicas, ratifica su veracidad y su pertinencia.

En este tenor, los dirigentes de la zona establecen como uno de los ejes referenciales claves en el proceso a dos médicos toxicólogos, quienes colaboraron con las Juntas de vecinos de las zonas afectadas, y no sólo emitieron informes para avalar las denuncias sino también "nos explicaron lo que nos pasaba”, haciendo uso del poder que en su calidad de expertos tenían de conformar y construir la experiencia de un cuerpo que duele, pero no tiene palabras para referir dicha experiencia. A partir del diagnóstico, se inauguró en los habitantes la posibilidad de denunciar y, con el tiempo, de ser reconocidos como "víctimas".

Si bien la importancia de la evidencia médica en la interpretación de la propia experiencia ayuda a la generación de "reclamo", produce un efecto problemático al interior de las comunidades. Al ser la prueba médica "positiva" uno de los hitos que "inaugura" la posibilidad de hablar por parte de los habitantes, tiende a constituirse también en un elemento que divide a la población entre "víctimas merecedoras" y "gente que se aprovecha". Así, las primeras se ubican entre quienes tienen exámenes con cifras altas de arsénico y plomo, mientras que la gente que no presenta estos niveles de contaminación es considerada "oportunista" en busca de beneficios estatales.

Esta división es problemática, pues los efectos de los desechos muestran bastante heterogeneidad en su distribución al interior del mismo territorio, incluso entre gente expuesta por la misma cantidad de tiempo y en el mismo cuadrante. ${ }^{8}$ Además, impide observar la condición transversal de vulnerabilidad que ha llevado al conjunto de los habitantes a estar expuestos a niveles de contaminación potencialmente perjudiciales para la salud. Así, paradójicamente, las Juntas de vecinos de las zonas afectadas y las familias hasta hoy en día terminan esperando que sus exámenes salgan positivos, porque esto

8 Según lo observado en los casos analizados, generalmente los efectos "comprobables" por exámenes de sangre no se distribuyen de manera homogénea en la población expuesta a niveles similares de contaminación, ya que el efecto de los agentes contaminantes interactúa con otros elementos como edad, alimentación, género, predisposición hacia algunas enfermedades, por ejemplo. En ese sentido, muchas veces los resultados sorprenden a los vecinos y ocasionan aún mayor especulación al no mostrarse de manera homogénea o al no aumentar con el paso del tiempo, lo cual la toxicología considera difícil de predecir o relacionar en un modelo de causalidad directa. 
constituye la única alternativa de ser escuchados y obtener alguna clase de reparación o "visibilidad".

La ratificación a través de los exámenes médicos del problema de la contaminación en la zona fue un elemento clave que activó a dichas Juntas y dio forma a las primeras acciones, tanto organizacionales como judiciales. Dentro de este proceso, destaca el rol del dirigente territorial Lombardo Molina y de las conocidas como "las catorce dirigentas del plomo", quienes dedicaron gran cantidad de tiempo a generar redes y a visibilizar el conflicto a nivel local.

Con estas acciones, el problema alcanzó cierta notoriedad pero no se establecieron medidas a nivel del gobierno central sino hasta mucho tiempo después. Como se señalaba anteriormente, la ratificación de las pruebas influyó de manera decidida en implementar el traslado de la parte más visible de los desechos tóxicos a la Quebrada Encantada. Sin embargo, el traslado se realizó sin asesoría técnica, y el polvo en suspensión, agravado por el contexto desértico, aumentó la dispersión de los desechos.

Pese a reconocer la peligrosidad de la situación, los habitantes siguieron viviendo en la zona. Frente a las escasas medidas precautorias, las acciones de presión de las Juntas de vecinos continuaron con "acampadas" en la plaza de la ciudad, marchas y manifestaciones que fueron transformando poco a poco sus subjetividades a esta idea de "víctima", "afectado" o sujeto "contaminado". A partir de este momento, el concepto de daño y reparación comenzó a formarse entre los vecinos.

\section{Medios de comunicación. Estar en la "tele" para ser "visible"}

Es posible ver un fenómeno similar respecto a los niveles de visibilidad pública desigual en relación con el segundo hito referencial: la intervención del programa de reportajes y denuncias Contacto, emitido en el año 2002 por la red de televisión abierta Canal 13.

Los periodistas de este conocido programa se interesaron por la historia de "las catorce dirigentas del plomo", quienes recientemente habían visitado la ciudad de Santiago para denunciar su problema ante la Presidencia con magros resultados. A los periodistas les llamó la atención lo poco público que era el conflicto e indagaron con base en información básica; las Juntas de vecinos les facilitaron un archivo elaborado por los mismos pobladores, el cual no sólo contenía documentos, exámenes médicos y catastro de la población enferma, sino también el archivo que guardaba la oficina de Medio Ambiente y que había sido entregado a estas dirigentas. 
Pese a que las dirigentes sociales habían utilizado los datos para hacer múltiples denuncias, hasta ese momento el problema seguía sin más intervención que el traslado de los restos a la Quebrada Encantada. A su vez, las organizaciones vinculadas con la denuncia de esta situación continuaban intentando introducir en la población la idea de que "algo no andaba bien", con resultados variables.

Con la información recopilada por los dirigentes de la zona y entrevistas in situ, los periodistas hicieron un programa que titularon "Contaminados" y que se emitió en un horario estelar por la red abierta. Dicha emisión fue decisiva en la acción de las autoridades centrales en la elaboración de un plan de intervención en la zona y para que los propios vecinos adquirieran un mayor conocimiento de su situación.

Las dirigentas recuerdan que después de años de solicitar reuniones sin éxito, una semana después del programa Contacto las autoridades centrales visitaron la zona y establecieron compromisos concretos de acciones al respecto. Así, el "Plan Maestro" es fruto de la controversia generada por dicho programa. Es en momento cuando los sujetos se volvieron realmente "visibles" para los distintos actores políticos a nivel nacional.

Cuando llegaron los niños del programa Contacto le dijimos: "No sé qué es lo que quieren, nosotros tenemos esto" y se lo tiramos arriba de una mesa en la casa de la señora Bety, teníamos unas cajas, unas cajas de plátanos llenas de cachureos, de papeles. Ellos quedaron muy asombrados, ellos dijeron, "ustedes acá tienen todo", "ustedes tienen todo", esas fueron palabras textuales. Ellos trabajaron con toda la información de nosotros, ellos revisaron punto por punto, se instalaron a vivir en la población. Y empezaron a investigar y bueno, yo creo que tú pudiste ver el programa y vas a ver que es un testimonio crudo (Dirigente territorial, mujer, marzo de 2014).

Si bien a partir de ahí se inaugura un periodo de grandes logros para las Juntas de vecinos en términos de ser escuchadas sus demandas, el impacto mediático del programa desató también un efecto inesperado y adverso: la estigmatización de la población y las reacciones de los habitantes de Arica al respecto. Las imágenes transmitidas buscaron aumentar el impacto, exacerbando la representación de una zona degradada y marginal, llena de gente enferma, basura y con altos componentes delincuenciales, lo cual repercutió directamente en el trato que los habitantes de estas zonas comenzaron a recibir de parte de quienes no pertenecían a la misma.

Al mostrar un territorio casi desconocido para algunos, no sólo se hizo público el problema, sino también se hizo pública y extendida la asociación entre pobreza, enfermedad y marginalidad con la población de dichos territorios. 
Yo quería prácticamente sacarme el estigma de polimetales, porque el estigma de polimetales acá en Arica es como que "el polimetales son las viejas rascas, ${ }^{9}$ son las viejas pobres, son las viejas de población, son Los Industriales, son Cerro Chuño", o sea, es lo más pobre de los más pobres es Polimetales, y más encima están contaminados y más encima están enfermos, es como, un perro sarnoso que nadie lo quiere (Dirigente territorial, mujer, junio de 2014).

Por otro lado, la emisión del Programa Contacto y su impacto mediático en la localidad también desató la competencia al interior de las organizaciones que se habían movilizado en torno a la denuncia de los sucesos. La figuración pública dividió a la vocería e instaló una competencia por el "capital político" que implicaban los líderes de un sector, si bien marginado, bastante numeroso en términos de padrón electoral.

Las alianzas, negociaciones y entrevistas dividieron a las organizaciones y provocaron rivalidades que se encuentran presentes hasta hoy en día en el territorio y que mostraron las características diversas de las poblaciones afectadas, independientemente de su condición compartida de vulnerabilidad. Los entrevistados indicaron explícitamente que desde este momento se perdió el carácter reivindicativo y se vincularon las vocerías a proyectos políticos de mayor alcance, lo cual en algunos casos específicos tuvo efectos positivos en términos de visibilidad pública, pero también generó relaciones clientelares en otros.

\section{Aparato jurídico en la configuración de experiencia tóxica}

El tercer hito también alude al reconocimiento por parte de una instancia externa al territorio y su influencia en la transformación de la experiencia tóxica de los habitantes de las zonas contaminadas. Este se refiere a la posibilidad del aparato jurídico de impactar en las subjetividades y reproducir posiciones desiguales, al ser una institución que conjuga la relación de poder cristalizada entre Estado y población vulnerable en términos de trato, con aquella relacionada con un conocimiento y experticia específica: el manejo de la ley, sus procedimientos y jerga asociada.

Un grupo de familias, lideradas por el dirigente Lombardo Molina, emprendió acciones judiciales contra el Estado, Promel y sus socios en Suecia en 1997. Luego de un largo proceso, la Corte Suprema determinó que la empresa sueca, dueña de los desechos tóxicos enterrados en la zona, debía reparar a las víctimas, además de hacerse cargo de una serie de medidas de mitigación. Se ordenó así indemnizar a 356 personas por $\$ 2.848$ millones de pesos (ocho

9 Término coloquial: pobre. 
millones por persona) por la responsabilidad que correspondía al Servicio de Salud de la Región, el cual, en representación del Estado chileno, debió haber tomado las precauciones del caso.

Esta reparación fue asignada sólo a quienes formaron parte de la demanda, por lo que no todos los habitantes afectados recibieran la compensación necesaria, lo cual dividió aún más a la comunidad. Sumado a esto, la reparación no implicó la relocalización de la población de la zona afectada: en el año 2009 la mayor parte de la población indemnizada permanecía ahí.

La reparación diferenciada dividió aún más a la comunidad, aunque sentó un precedente clave para ratificar la situación ante los ojos de los vecinos incrédulos. Muchos de ellos refieren que fue en este momento cuando se vincularon al movimiento de protesta, bajo la idea de que "si los suecos pagaron, por algo será”. La peligrosidad de los desechos sólo adquiere realidad entonces para algunos, al existir una entidad externa, legítima, poderosa, dispuesta a reconocer y reparar aunque sea de manera mínima a los afectados.

El año 2007 salió la demanda de los polimetales, salieron ganadores trescientas ochenta y tantas personas, no me acuerdo el número fijo, ahora en este momento. Pero la demanda llevaba mil y tantas personas, era la demanda del año 98. Entonces cuando salió esa demanda, yo debo reconocerlo, dije: "Oye, ¿por qué ciertas personas ganaron?, tiene que haber un daño" (Dirigente territorial, mujer, junio de 2014).

Una vez conseguida la primera victoria, en el territorio proliferaron iniciativas legales. Muchos de los entrevistados apenas recuerdan cuántas demandas han firmado, dirigidas a quién y con qué efectos. El rol de los "abogados" en este contexto se vuelve una figura central en el fortalecimiento de la experiencia de vulnerabilidad e incertidumbre de los habitantes: los abogados "aparecen" y "desaparecen" en el territorio, muchos de ellos no vuelven a visitarlos ni contestan las llamadas de los dirigentes de las organizaciones.

En una dinámica también centrada en el poder del conocimiento experto, muchos de los habitantes estaban involucrados en las casi 13 demandas colectivas que, según se pudo constatar en tribunales, habían sido abandonadas por el abogado a cargo al ser objetadas en una primera instancia por errores propios del mismo profesional. Pese a este abandono de más de siete años, el abogado jamás señaló esto a quienes formaban parte de dichas acciones ni contestó sus llamadas.

Tan es así, que durante la primera fase de esta investigación realizada en el año 2014, los relatos de los sujetos refieren a causas "aún" vigentes y se encuentran esperando resultados en torno a ellas con gran expectación. Durante la estancia de terreno de enero de 2015 algunos de los involucrados recién habían sido notificados por un nuevo abogado a cargo de que sus 
causas estaban archivadas y que muchos de ellos, al haber presentado ya una acción judicial, no podrían emprender nuevas acciones por el problema de la proscripción de ciertos delitos. La sensación de los entrevistados era de amargura y resignación por la situación, tras casi diez años de espera.

De esta manera, el elemento judicial se constituyó en un punto clave para la articulación de las organizaciones ejes del conflicto y la búsqueda de reparación. Según lo narrado por los actores y durante el trabajo etnográfico, se pudo constatar que buena parte de las gestiones de los dirigentes estaban articuladas en torno a la generación de estas demandas y a la posterior localización de los abogados para obtener información respecto a los resultados.

En este proceso se jugaba también el "capital político" de los dirigentes sociales frente a otro considerado casi como un "superior", al cual no se puede acudir directamente: el abogado. Al dirigente se le solicitaba el rol mediador -el "que nos explique con peras y manzanas ${ }^{10 "}$ - entre el abogado y la comunidad, para que informara exactamente y en términos más cotidianos qué sucedía con el tema legal, qué documentos y papeles se necesitaban, entre otras cosas.

Si los dirigentes no podían dar información al respecto, los vecinos consideraban que no eran "eficientes", "ocultaban cosas" o no tenían la capacidad para llegar a los abogados. Por eso, gran parte del actual descrédito que tienen algunos dirigentes en el territorio -concretamente las conocidas como "las catorce dirigentas del plomo"- se asocia al destape de la situación de las demandas que nunca llegaron a finalizarse y que fueron gestionadas por estas personas a nivel de territorio, a pesar de que éstas tampoco tuvieron ninguna capacidad real de presión sobre el abogado que abandonó las causas.

En ese sentido, uno de los efectos inesperados y ciertamente negativos sobre la importancia dada a la judicialización como mecanismo para obtener reparación ha sido el descrédito de la acción colectiva en torno al logro de objetivos comunes. El escaso éxito de las acciones judiciales emprendidas por las Juntas de vecinos, sumadas a la incertidumbre e información contradictoria en torno al proceso de construcción de la demanda y finalización, provocaron que los habitantes tuvieran la percepción de que la permanencia en la organización no sólo había sido inútil, sino hasta perjudicial.

Así, luego del auge del conflicto en años anteriores, actualmente los pobladores se encuentran en una fase de desesperanza y/o acción individual, así como de profunda desconfianza hacia las organizaciones territoriales. Este hecho se vio reforzado cuando a finales del año 2014 la Corte Suprema admitió una demanda realizada de manera individual por una familia, indemnizando el Servicio de Salud Regional a cuatro personas.

10 Expresión coloquial: Que explique en términos simples y sencillos. 
Los beneficiarios de este veredicto constituyen un caso paradigmático de una salida individual beneficiosa que aumentó la amargura de quienes invirtieron su tiempo en la acción colectiva: estos vecinos no tuvieron extensa relación con nadie del territorio, nunca quisieron involucrarse en las organizaciones ni en las acciones de protesta y contrataron a un abogado de manera individual para que llevara su causa. En lugar de esperar las medidas de erradicación, acudieron al Servicio de Vivienda para que permutara su casa en la zona contaminada por otra en una zona rural aledaña a Arica.

Lo mediático del fallo favorable a esta familia golpeó fuertemente a las organizaciones vecinales que, durante ese mismo periodo, llevaban casi diez años esperando sus causas en tribunales y que recién estaban empezando a saber que éstas se encontraban abandonadas. El efecto inmediato de esto fue la gran cantidad de causas individuales que llegaron a manos del abogado victorioso en menos de dos meses, incluyendo las de dirigentes emblemáticos del territorio.

Respecto al caso puntual de la demanda de 1997 que ratificó la percepción de los sujetos de que "algo andaba mal", la reparación no tuvo el efecto esperado por quienes la cursaron: muchos de los que tuvieron acceso a esta reparación gastaron sus recursos ampliando o mejorando las casas en los sitios contaminados, lo cual da cuenta de la escasa noción de la peligrosidad de la contaminación de la zona o, como nos refirieron algunos de los afectados, de que el monto no estaba pensado para que se pudieran mover, pues no les alcanzaba para trasladarse a otra vivienda.

Podemos ver que el aparato judicial también tiene una gran importancia en la experiencia tóxica y en el sufrimiento de los habitantes. Por una parte, los coloca en una situación de conocimiento desigual frente a "otro" abogado que monopoliza la posibilidad de obtener justicia; y, por otra, cuando se obtiene esta reparación, no sólo le pone un precio al dolor ocasionado -que muchas veces no se condice con el daño causado y el cual aún falta por observar en el largo plazo-, sino también pone en marcha procesos que generan nuevas formas de victimización de la población por su condición vulnerable.

Un ejemplo claro de esto es el tratamiento de la causa reciente ganada en la Corte Suprema de manera individual por la familia Sanhueza, en el que la desacertada acción del poder judicial en lugar de reparar, ha infringido nuevas formas de victimización sobre esta familia: luego de casi seis años de litigio por la grave enfermedad de sus dos hijos pequeños, se asignó una reparación de diez millones de $\operatorname{pesos}^{11}$ por cada miembro del grupo familiar, pero al transcribir el fallo formalmente y realizar la orden de pago, los funcionarios

11 US=16 008, al 9 de marzo de 2015. 
del poder judicial cometieron un error que redujo el pago a sólo diez millones de pesos en total, sin posibilidad de réplica ni apelación a pesar de que evidentemente se trata de un error de transcripción de acuerdo con los documentos oficiales. Esta información les fue entregada a los afectados luego de mucho presionar en los tribunales en busca de una explicación.

Junto con la incertidumbre de no saber qué sucedió, en el momento de escribir este artículo el demandante había hecho gestiones en casi todas las instancias municipales, regionales y nacionales para aclarar la situación sin éxito, utilizando gran parte de su tiempo en largas esperas frente a las oficinas de diversos funcionarios quienes le aconsejaban "aceptar lo que hay, porque es mejor que nada”.

La impotencia ante el abuso reiterado que sufrían golpeó emocionalmente con dureza a esta familia, según relataron sus integrantes cuando se les visitó en el marco del trabajo de campo, lo cual constituyó también, sin duda, uno de los momentos emocionalmente más intensos para la investigadora a cargo de documentar su experiencia. ${ }^{12}$

\section{Intervención estatal. Politica pública, reparación y revictimización}

Como es posible ver, en cada uno de los puntos anteriores es posible distinguir posibilidades para la reparación y la acción colectiva, pero también nuevas formas de victimización de la población. Ambos elementos pueden ser observados también en las políticas públicas que se han implementado en el territorio. Las principales fueron las medidas del "Plan Maestro" y la posterior "Ley de Polimetales", actualmente en ejecución. Los problemas en la implementación de dichas medidas vienen de mucho antes de su puesta en marcha, sobre todo en la errática y deslegitimada acción de los actores institucionales y la escasa competencia técnica para lidiar con la complejidad de la situación.

Antes de que existiera el documento que dio forma al "Plan Maestro", los habitantes del territorio ya manifestaban su desconfianza frente a la acción de algunos actores, quienes una y otra vez habían intentado ocultar la situación:

Las autoridades siempre nos dijeron que no estábamos contaminados, siempre nos engañaron y se confabularon. Eso es lo más triste, se confabularon, se confabularon con la Universidad, la Universidad cambió nuestros exámenes. Acá tuvo que ver mucho el

$12 \mathrm{Al}$ respecto, no cabe más que agradecer de manera especial a la familia Sanhueza por haber recibido a nuestro equipo en su casa y haber compartido experiencias sumamente dolorosas con quienes éramos unos desconocidos para ellos. Esperamos contribuir, al menos mínimamente, a visibilizar su problema y a que puedan encontrar reparación. 
tema de que fuera personas con escasos recursos, de hecho, nos costaba incluso llegar a Santiago, estamos a dos mil kilómetros, el viaje en bus era terrible, con suerte tal vez nos conseguíamos algunas veces pasaje en avión. Que nos escucharan fue la pelea más dura de la vida yo creo (Dirigente territorial, mujer, junio de 2014).

Junto con esta falta de legitimidad, durante varias de las intervenciones las autoridades competentes no tuvieron la adecuada asesoría técnica para lidiar con un caso tan complejo; mientras éste no adquirió relevancia nacional, no se hizo un esfuerzo real por instaurar medidas adecuadas técnica y humanamente.

Pese a la dificultad de establecer los territorios afectados por la dispersión aérea de los desechos, se determinaron zonas críticas - "El Polígono"- sobre las cuales se focalizó la intervención, lo cual constituyó una fuente de permanente conflicto e incertidumbre para la población, ya que la delimitación de zonas críticas incluyó en un principio un radio bastante menor en relación con lo solicitado por las organizaciones vecinales y tuvo que ser modificado en varias ocasiones.

Muchas de las zonas afectadas con dirigentes territoriales emblemáticos quedaron fuera de "El Polígono" en un primer momento, sin saber si recibirían ayuda aun cuando se encontraban a pocos metros de la zona considerada como "peligrosa". Según los datos del Censo 2002 disponibles al momento de la intervención del "Plan Maestro", en las zonas afectadas residían alrededor de 12.660 personas, quienes habitaban 3.752 viviendas.

Sin embargo, se consideró que la población afectada era de 5.000 personas y sobre este número se aplicaron las medidas, las cuales no sólo eran insuficientes sino que fueron implementadas de manera heterogénea por cada sector. Al hablar con los encargados locales de su aplicación, señalaron que la heterogeneidad de las medidas se debía a los requerimientos de los mismos sectores o dirigentes de las organizaciones de cada lugar, a la hora de implementar el conjunto de soluciones establecidas en el Plan Maestro.

Con estas prácticas se fomentó la atomización de las organizaciones, la división entre los vecinos y lo que es peor, no se tomaron las medidas técnicamente necesarias para proteger a la población completa. Entre dichas medidas se consideraba la pavimentación rápida de los sectores afectados para evitar la dispersión por aire, la ampliación de estudios para conocer la profundidad de la presencia de metales y la "relocalización de viviendas ubicadas en el Sector F, que incluyen las calles Renato Rocca, Alejandro Azola, Capitán Avalos, Cerro Chuño, Calles 1, 2, 3, 4, 7, 8 y Mario Ojeda, las cuales corresponderían a aproximadamente 1880 viviendas"13 (Programa Maestro, 2009: 26).

13 Estas definiciones se modificaron en el marco de la implementación de la ley, incluyéndose sectores antes no considerados como de riesgo, a partir de la acción y reclamo de los vecinos. 
Se propuso también la implementación de un plan de salud que consideró la atención en la red primaria estatal de los afectados y se elaboró un sistema de acompañamiento de los procesos de aprendizaje de los niños en las escuelas de las zonas afectadas, entre otras medidas. Pero la puesta en marcha de éstas, ha conllevado una serie de problemas que han impuesto un nuevo sufrimiento a los sujetos. El primero es la lentitud: se puede rastrear el inicio de las denuncias a mediados de la década de 1990, mientras que el primer "Plan Maestro" corresponde a 2009 y la ley que permite su implementación data del 2012.

Considerando que a la promulgación de la ley se le acompañó también la elaboración de un "reglamento" destinado a definir los aspectos operativos, los beneficios y políticas reparatorias establecidos en la ley comenzaron a llegar al territorio a partir de 2013. Tiempos cortos en términos del aparato estatal, pero largos en relación con la salud de la población.

Durante ese periodo, las organizaciones se fraccionaron varias veces, los vecinos que tenían la posibilidad de irse simplemente abandonaron las viviendas en el polo afectado y éstas fueron tomadas por nuevos ocupantes, en su mayoría migrantes indocumentados, vagabundos o familias en extrema pobreza. Las zonas donde se debían mitigar los daños presentan escaso avance: en algunos casos se ha limpiado el polvo de los techos y se han creado "plazas secas". ${ }^{14}$ Sin embargo, poco se ha avanzado en la pavimentación total de las calles y de los patios de las viviendas, donde aún hay tierra contaminada, a la cual se expone la población diariamente.

Una segunda fuente de sufrimiento por las políticas reparatorias proviene de las medidas de relocalización de los habitantes de zonas que estaban definidas como críticas en la ley. Como primer punto, cabe señalar que han sido lentas e inefectivas, ya que buena parte de las familias que debieron ser trasladadas permanecen en la zona afectada; sólo parte de Cerro Chuño ha sido beneficiario de la relocalización.

Como segundo punto, en la zona donde esta medida de erradicación ha sido efectivamente cumplida, se ha implementado con diferencias en los tiempos y en la calidad de las casas entre vecinos con el mismo tipo de vivienda. ${ }^{15}$ Esto ha ocasionado conflictos e insatisfacción entre la gente, aumentando la sensación de que el Estado ofrece soluciones de "o lo tomas o lo dejas" y de incertidumbre en los casos de quienes no aceptaron las primeras condiciones de reubicación.

14 Espacios recreacionales completamente sellados por pavimento, sin pasto ni tierra.

15 A unos se les han entregado departamentos, a otros, casas, dependiendo de la disponibilidad de recursos del Ministerio de Vivienda. 
Por otro lado, llevar a cabo por etapas la relocalización también ha implicado que quienes se han ido, han abandonado viviendas sin agua, sin luz, las cuales se encuentran actualmente tomadas por población en situación de calle; esto aumenta la tensión entre los vecinos, los delitos y la degradación del barrio.

Cuando se estaba escribiendo el presente artículo, se iba a producir una segunda "oleada" de relocalizaciones en Cerro Chuño, y las familias que permanecerían en la zona esperando una solución para finales de 2015 -alrededor de 50 viviendas- temían por su seguridad en un contexto donde ya no existe transporte público ni comercio, donde la basura se acumula por los rincones y la mayor parte de las viviendas, pese a su clausura, estaba habitada ilegalmente. Todo esto sin contar que los nuevos hogares entregados se hallan a escasos kilómetros de "El Polígono" contaminado.

Tanto en el caso de las otras poblaciones relocalizadas como en el de las que aún permanecen a la espera, existen tensiones constantes respecto a que las viviendas ofrecidas como solución por el Ministerio de Vivienda no tienen características similares a las que los afectados poseían, ni compensan las inversiones hechas en su mejora, lo cual constituye una tercera fuente de sufrimiento.

En lugar de adoptar una posición que propiciara el diálogo y la reparación de los afectados, -criterio que debería primar en la responsabilidad que le compete al Estado por el daño ocasionado-, se otorgaron viviendas según el presupuesto disponible y no en función de las necesidades y demandas mínimas de las organizaciones y habitantes. Se cambiaron casas pequeñas por departamentos que no tenían la misma superficie, ni el valor ni ventajas en términos de ubicación que los de sus casas originales.

Por esta razón, la sensación de "perder el esfuerzo de tantos años" y "empezar de cero" hizo que algunos de ellos rechazaran la oferta de relocalización y prefirieran permanecer en las zonas afectadas. Por lo demás, la ubicación de las nuevas viviendas a escasos metros de "El Polígono" contaminado despertó desconfianza entre los vecinos y los instó a permanecer en las suyas, aun cuando muchos enviaron a sus hijos a vivir a otros lugares. Todos estos elementos han ocasionado que el conflicto se mantenga vigente, acrecentando la percepción de abuso y vulnerabilidad en los habitantes. 


\section{Conclusiones}

El caso presentado muestra los procesos conflictivos de construcción de sentido en torno a la experiencia de vivir en un ambiente degradado. De esta manera es posible ver cómo la construcción de los relatos producidos en este contexto se encuentra estrechamente vinculada con las relaciones de dominación que cruzan los territorios y delimitan quiénes son los actores autorizados y legítimos para hablar, cómo y cuándo.

A partir de estas relaciones, los sujetos van elaborando su "gramática de la desigualdad” (Boltanski y Thévenot, 1999), en un juego constante entre la victimización y la construcción de sentido desde los intersticios existentes entre el coro de voces provenientes de los distintos actores y les dicen cómo interpretar su propia experiencia. Es en estos intersticios donde los afectados van encontrando su propia voz, uniendo la información disponible de manera creativa y estableciendo así su propio marco de sentido (Butler, 2010) para orientar su acción en las circunstancias en que se encuentran.

Dentro de estas voces, una de las más importantes es la del "experto", quien aporta la evidencia empírica y médica capaz de constituir al sujeto víctima, dar forma a la experiencia corporal que implica vivir en un contexto tóxico y entregar herramientas para "entender lo que nos pasa". El momento de la prueba médica es fundamental e inaugural tanto en la constitución de subjetividades como para instalar la noción de daño y, por ende, la idea de reparación y responsabilidades. Luego de este momento inicial, el proceso de obtención de reconocimiento va dejando huellas en los sujetos y depende también de los distintos actores que van moldeando estas experiencias tóxicas: el Estado, la prensa, el poder judicial.

En dichos procesos de "reconocimiento", los sujetos se ven involucrados en relaciones desde una posición de extrema vulnerabilidad, por la intersección con otras variables como los niveles de pobreza y desprotección social, el género, los niveles de participación y visibilidad pública desigual. Los habitantes del caso de Polimetales en Arica deben disputar esta visibilidad desde el lugar de quienes ya son invisibles: los usuarios de vivienda social, los habitantes de los márgenes urbanos. En ese sentido, la experiencia de vivir en un ambiente degradado agudiza su violencia con la intersección de ejes de desigualdad operando en un mismo territorio, marcado por flujos y actores políticos y económicos cuyo alcance supera por mucho la acción de sus habitantes.

Finalmente, y a la luz de estos casos, me gustaría aportar en torno a por qué resulta importante introducir la dimensión socioecológica en los análisis de desigualdad y conflicto. En primer lugar, la experiencia de desigualdad 
socioecológica es relativamente nueva en relación con otras variables de desigualdad persistente que han estructurado nuestras historias nacionales. Si bien la disputa por el acceso y distribución de los beneficios de la explotación de recursos naturales es de larga data, el debate sobre los efectos negativos de estas actividades no tiene más de treinta años de visibilidad en el caso chileno. En ese sentido, es precisamente acá donde es posible ver arme y desarme de los mecanismos de legitimación/ tolerancia o conflicto en torno a las desigualdades.

En segundo lugar, las desigualdades socioecológicas resultan particularmente violentas para los sujetos, pues generan efectos visibles e impactan muchas veces en el corto plazo en la biografía y subjetividades de los individuos, al tener consecuencias sobre los cuerpos, la salud y la forma de pensar los espacios colectivos. Por ello se vuelve especialmente relevante documentar los aspectos cualitativos asociados con estos procesos, para comprender cómo es la experiencia de vivir en las sociedades desiguales más allá de las cifras y entender a quien habita en ellas como el sujeto que es hoy protagonista de nuestras democracias.

Finalmente, me gustaría destacar que este es un fenómeno en expansión, ligado precisamente a la concentración urbana y al auge renovado del extractivismo en América Latina, el cual se acrecentará en la próxima década. En el marco de esas tendencias y dados los niveles de inequidad en nuestras sociedades, no podemos sino esperar una distribución fuertemente desigual de los niveles de riesgo y el aumento del sufrimiento ambiental, con efectos desconocidos sobre las comunidades en el largo plazo, que requieren de mecanismos de protección, regulación e intervención.

\section{Referencias}

Acker, Joan (2006), “Inequality Regimes: Gender, Race, and Class in Organizations”, en Gender \& Society, vol. 20, núm. 4, EUA: Oakland University.

Aguirre, L. (2011), "Desigualdades, racismo cultural y diferencia colonial", en Working Paper, núm. 5, Berlín: Desigualdades.Net.

Araujo, Kathya (2009), Habitar lo social. Usos y abusos en la vida cotidiana del Chile actual, Santiago de Chile: LOM.

Araujo, Kathya y Darío Martucelli (2011), "La inconsistencia posicional: un nuevo concepto sobre la estratificación social”, en Revista CEPAL, núm. 103, Santiago de Chile.

Auyero, Javier y Débora Swistun (2008), Inflamable. Estudio del sufrimiento ambiental, Buenos Aires: Paidós.

Baztan, Ángel (1995), Etnografía. Metodología cualitativa en la investigación sociocultural, Ciudad de México: Alfaomega.

Bertaux, Daniel (1997), Pathways to Social Class: a qualitative approach to social mobility, Oxford: Clarendon Press. 
Convergencia Revista de Ciencias Sociales, núm. 72, 2016, Universidad Autónoma del Estado de México

Boltanski, Luc et al. (2006), On Justification: Economies of Worth, New Jersey: Princeton University Press.

Boltanski, Luc y Eve Chiapello (2002), El Nuevo Espíritu del Capitalismo, Madrid: Akal.

Boltanski, Luc y Laurent Thévenot (1999), "The sociology of critical capacity”, en European Journal of Social Theory, núm. 2, Sussex: Sage Publications.

Byrne, D. (2005), "Class, Culture and Identity: A Reflection on Absences Against Presences", en Sociology, vol. 39, núm. 5, Londres: Sage.

Bullard, Robert (1990), Dumping in Dixie: Race, Class, and Environmental Quality, Boulder, San Francisco, Oxford: Westview Press.

Bullard, Robert (1999), Confronting Enviromental Racism. Voices from the Grassroots, Cambridge: South End Press.

Bullard, Robert (2005), The quest of for the enviromental justice. Human Rights and the politics of pollution, San Francisco: Sierra Club Books.

Butler, Judith (2010), Marcos de guerra. Las vidas lloradas, Barcelona: Paidós.

Castillo, Juan C. (2009), “Cuál es la brecha salarial justa? Opinión pública y legitimación de la desigualdad económica en Chile”, en Estudios Públicos, núm. 113, Santiago: Centro de Estudios Públicos.

Castillo, Mayarí (2013), "Construyendo categorías para pensar la agencia política en sociedades desiguales. Una reflexión sobre Arendt y Butler”, en Revista Internacional de Pensamiento Politico, núm. 7, España: Universidad Pablo de Olavide y Universidad de Huelva.

Castillo, Mayarí (2015), “Desigualdades socio ecológicas en Chile contemporáneo. Legitimación y conflicto en torno al sufrimiento ambiental”, en Desigualdades, Legitimación, Tolerancia y conflicto en las Sociedades Latinoamericanas, Santiago, Chile: RIEL Editores, Center for Cohesión and Social Conflict (COES), ICIIS, Desigualdades.net.

Cole, Luke y Sheila Foster (2001), From the Ground Up: Enviromental Racism and the rise of the environmental justice, Nueva York: New York University Press.

Costa, Sergio (2011), "Researching Entagled Inequialities in Latin America. The Rol of Historical, social and Transregional interdependencies", en Desigualdades Working Papers, núm. 9, Berlín: Freie Universität Berlin.

Chaterjee, Partha (2008), “Grupos de población y sociedad política”, en La nación en tiempo heterogéneo,Buenos Aires: CLACSO, Siglo XXI.

Checker, Melissa (2005), Polluted Promises. Enviromental Racism and the Search for Justice in a Southern Town, Nueva York: New York University Press.

Dosbon, Andrew (1998), Justice and the Environment: Conceptions of Environmental Sustainability and Theories of Distributive Justice, New York: Oxford University Press.

Elvers, Horst-Dietrich (2007), "Umweltgerechtigkeit als Forschungsparadigma der Soziologie”, en Soziologie, vol. 36, núm. 4, Berlín: Verlag für Sozialwissenschaften.

Fuenzalida, Manuel y Rodolfo Quiroz (2012), "La dimensión espacial de los conflictos ambientales en Chile”, en Polis, Revista Latinoamericana, vol. 11, núm. 31, Santiago de Chile: América en Movimiento.

Gootenberg, Paul y Luis Reygadas [eds.] (2010), Indelible inequalities in Latin America: insights from history, politics, and culture, Durham y Londres: Duke University Press.

Gootenberg, Paul y Luis Reygadas (2004), "Desigualdades persistentes en América Latina. Historia y Cultura”, en Alteridades, vol. 14, núm. 28, México, D.F.: UAM Iztapalapa. 
Guimarães, Roberto (2012), "Environment and Socioeconomic Inequalities in Latin America”, en Notes for a Research Agenda Working Paper, núm. 20, Berlín: Iberoamikanishes Institut.

Harvey, David (1996), Justice, Nature and the Geography of Difference, Malden, Massachusetts: Blackwell.

Instituto Nacional de Estadística (INE) (2002), Censo Nacional de Población. Disponible en: http://www.ine.cl/cd2002/ [01 de agosto de 2016].

McCall, Leslie (2005), “The complexities of intersectionality”, en Signs, vol. 30, núm. 3, Chicago: University Chicago Press.

Ministerio de Desarrollo Social, Gobierno de Chile (2006), Encuesta de Caracterización Socioeconómica Nacional. Disponible en: http://observatorio. ministeriodesarrollosocial.gob.cl/casen/casen_usuarios.php [01 de abril de 2014].

Müller, Markus y Susan Clayton (2013), "Introduction to "Environmental Justice”, en Social Justice Research, septiembre, vol. 26, núm. 3, New York: Springer.

Organización Mundial de la Salud (2012), "Arsénico", en Nota Descriptiva, núm. 372, diciembre. Disponible en: http://www.who.int/topics/arsenic/es/ [1 de agosto de 2016].

Pezzullo, Phaedra (2001), "Performing Critical Interruptions: Stories, Rhetorical Invention, and the Environmental Justice movement", en Western Journal of Communication, vol. 65, núm. 1, Murfreesboro.

Programa Maestro de Intervención Zonas con Presencia de Polimetales en Arica (2009), Arica, Chile: Gobierno Regional.

Reichards, Michael (2003), Poverty Reduction, Equity and Climate Change: Global Governance Synergies or Contradictions?, London: Overseas Development Programme.

Reygadas, Luis (2004), "Redes de la desigualdad. Un enfoque multidimensional”, en Politica y Cultura, núm. 22, México D.F.: UAM Xochimilco.

Reygadas, Luis (2008a), "Distinción y reciprocidad. Notas para una antropología de la equidad", en Revista Nueva Antropología, vol. XXI, núm. 69, Ciudad de México.

Reygadas, Luis (2008b), La apropiación. Destejiendo las redes de la desigualdad, Barcelona: Anthropos.

Savage, Mike (2001), Class Analysis and Social Transformation, Philadelphia: Open University Press.

Savage, Mike (2008), "Affluence and Social Change in the Making of Technocratic Middle Class Identities”, en Contemporary British History, núm. 22, vol. 4, London: Routledge.

Savage, Mike et al. (2008), "Class and Cultural Division in the UK", en Sociology, Núm. 42, vol. 6, London: Sage Publications.

Smith, Neil (2008), Uneven Development. Nature, capital and the Production of Space, Atenas y Londres: The University of Georgia Press.

Tilly, Charles (2000), La desigualdad persistente, Buenos Aires: Manantial.

Thévenot, Laurent et al. (2011), "An interview with Laurent Thévenot: on engagement, critique, commonality and power", en European Journal of Social Theory, vol. 14, núm. 383, Sussex: Sage Publications.

Thévenot, Laurent (2007), “The Plurality of Cognitive Formats and Engagements Moving between the Familiar and the Public", en European Journal of Social Theory, vol. 10, núm. 3, Sussex: Sage Publications. 
Vásquez, Alexis y Marcela Salgado (2009), “Desigualdades socioeconómicas y distribución inequitativa de los riesgos ambientales en las comunas de Peñalolén y San Pedro de la Paz. Una perspectiva de justicia ambiental", en Revista de Geografía Norte Grande, núm. 43, Santiago de Chile.

Wilkinson, Richard (2005), The Impact of Inequality: How to Make Sick Societies Healthier, Nueva York: Routledge.

Wright, O. Erik (1985), Classes, London: Verso.

Wright, Erik O. (2009), "Understanding Class. Towards an Integrated Analyttical Approach", en New Left Review, nú m. 60, Londres.

Mayarí Castillo Gallardo. Antropóloga Social, Universidad de Chile. Maestra en Ciencias Sociales, FLACSO México. Doctora en Sociología, Freie Universität Berlin. Académica de la Escuela de Antropología de la Universidad Academia de Humanismo Cristiano, investigadora del Comparative Research Programme on Poverty (CROP) y del Centro de Estudios Interculturales e Indígenas (CIIR). Líneas de investigación: estratificación, desigualdad y pobreza; conflictos socioambientales y urbanos. Publicaciones recientes: Mayarí Castillo, "Socio-ecological inequality and water crisis. Views of indigenous communities in the Alto Loa area", en Environmental Justice, febrero, vol. 9, núm. 1, Mary Ann Liebert Publishers (2016); Mayarí Castillo, "Fronteras simbólicas y clases medias. Una aproximación cualitativa al proceso de movilidad social en Chile contemporáneo", en Revista Perfiles Latinoamericanos, núm. 48, Facultad Latinoamericana de Ciencias Sociales (2016); Mayarí Castillo y Claudia Maldonado, Desigualdades. Legitimación, Tolerancia y conflicto en las Sociedades Latinoamericanas, Santiago, Chile: RIL Editores, The Center for Cohesión and Social Conflict (COES), ICIIS (2015).

Recepción: 28 de abril de 2015.

Aprobación: 6 de julio de 2016. 